\title{
Bony Exostoses on Hard Palate - Prosthetic Inferences
}

\author{
Shuja Rahman', Anchal Deep ${ }^{2}$, Khurshid A Mattoo ${ }^{3 *}$ \\ ${ }^{1}$ Department of Prosthodontics, Kothiwal dental college, MJPR University. \\ ${ }^{2}$ Department of prosthodontics, Ideas dental college, MPMS University. \\ ${ }^{3}$ College of dentistry, Jazan University, Saudi Arbia. \\ drkamattoo@rediffmail.com \\ *Corresponding author: Khurshid A Mattoo, College of dentistry, Jazan University, Saudi Arbia.
}

\begin{abstract}
Bone is a vital tissue and responds to mechanical stresses in the form of deposition or resportion depending upon the intensity of the force. Excess bony growth in the oral cavity can hamper functions of oral cavity, especially phonetics and affect fabrication of a dental or orthodontic prosthesis. We report a case of a 42 year old patient, who presented with a diffuse bilateral bony exostoses in the region of the posterior hard palate. Besides academic interest, such bony variation has prosthetic implications which are also discussed. The patient's chief complaint was to restore an endodontically treated mandibular left first molar which was restored with a metal ceramic single crown.
\end{abstract}

Keywords: compact bone, torus mandibularis, complete denture, fixed partial denture.

\section{INTRODUCTION}

Exostoses and tori, is a term well known in anthropology since the publication of the first article by Gox in $1814 .{ }^{1}$ The two names are interchangeable and are given to the bony protuberances arising from various bony surfaces of the maxilla or mandible. Having a multifactorial etiology, they are routinely discovered during dental treatment and pose no difficulties whatsoever to the patient unless the size is excessive. In such cases, they may pose functional, esthetic and psychological fear in patients. Depending upon the location and their number, ${ }^{2}$ they are termed tori or exostoses like torus palatinus, torus mandibularis, buccal exostoses and palatal exostoses. Histologically both share characteristic typical features of a hyperplastic bone that comprises of mature trabecular and cortical bone. ${ }^{3}$ Torus palatinus and torus mandibularis have been reported commonly. We report a case of bilateral palatal exostoses on a very rare location on the posterior palatal surface of the maxillary tuberosity. Various prosthetic implications related to both removable and fixed prosthodontics are also discussed.

\section{CASE REPORT}

A male patient aged 42 years, was referred from department of oral medicine and diagnosis to the department of prosthodontics for placement of a single crown in relation to an endodontically treated mandibular left sided first molar. Patients medical, social and drug history were non relevant to the existing treatment. The patient was a chronic pan chewer and smoker and used to consume "Thara" a form of local brewed alcohol in North India. The frequency of consumption was once/twice a week depending upon the availability of the money. Extra oral examination did not disclose any significant negative findings while intra oral examination presented a picture of extrinsic staining of teeth,

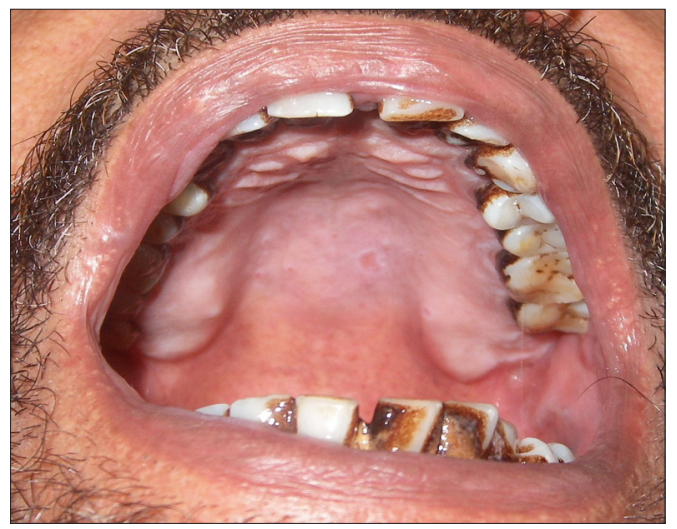

Fig 1. Maxillary posterior palatal region showing bilateral diffuse bony outgrowths extending up to the first molar region 
generalized plaque and calculus deposits, arrested pit and fissure caries in a few posterior teeth. Mandibular anteriors showed severe calculus deposits. In the maxillary arch, there was a bilateral expansion of the cortical bone that started from the second molar and ended near the pterygoid hamulus (Fig 1). On either side the bony projection was smooth and presented with a mild undercut towards its posterior end (1to 2 millimeter). The bony expansion was diffuse and merged well with the maxillary tuberosity on either side. Since the presence of bony expansion were non contributory, the treatment plan included oral hygiene maintainence followed by the fabrication of a porcelain fused to metal (buccal facing) crown in relation to right mandibular first molar. Diagnostic preliminary impressions were made of irreversible hydrocolloid (Fig 2) to assess the palatal exostosesRoutine clinical and laboratory procedures were followed for the fabrication of the single crown in relation to endodontically treated mandibular left first molar. The patient was put on a follow up protocol that lasted for a year during which the patient disclosed to have no complaints regarding the functioning of the single crown.

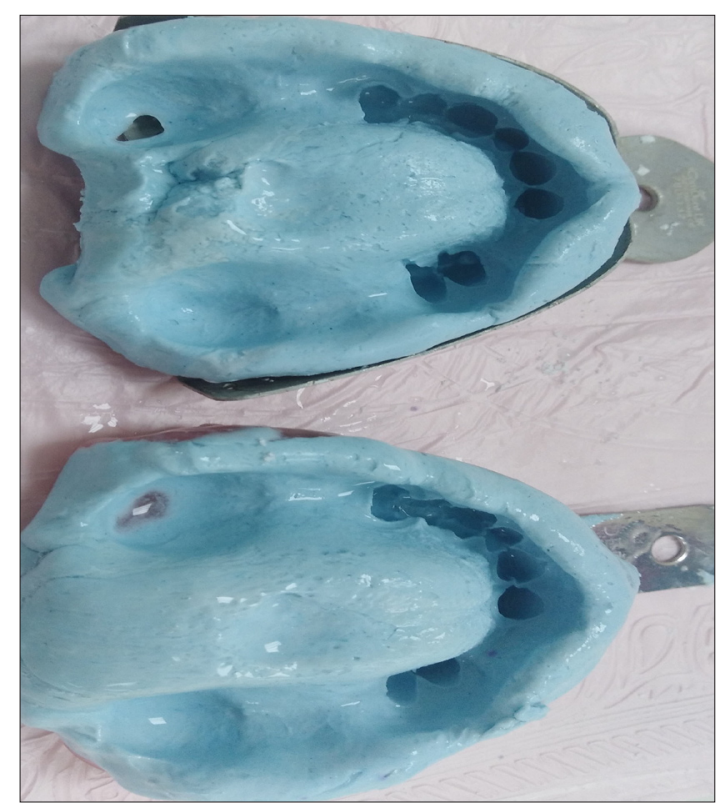

Fig 2. Diagnostic impressions showing bilateral bony growths (depressed areas) in the posterior region

\section{Discussion}

Dental surgeons often encounter abnormal variations in hard and soft tissue within the oral cavity and most of these variations are discovered routinely while fabricating a dental prosthesis. ${ }^{4}$ Posterior palatal anatomical region is very important for prosthodontist especially during removable prosthesis fabrication. The abnormalities in the posterior palatal area can be either congenital of acquired and may involve only soft or hard tissue or both. ${ }^{5}$ We present a case of bilateral bony exostoses of a 42 year old patient. The case is unique since bony exostoses are usually multiple in number and are normally never symmetrical. In this case, we have a single bony growth on both side and nearly identical and symmetrical in size, shape and extent which is very rare. The etiology of bony tori/exostoses is multifactorial that involves genetic, environmental and host tissue response factors. The theory that explains the etiology of tori and exostoses, is continuous genetic or threshold theory. ${ }^{6}$ Correlation between parafunction and the existence of tori has been demonstrated along with dietary habits and nutritional disturbances. ${ }^{7}$ However, no single factor is conclusively associated with its cause. Depending upon the location, size, extent, morphology and relation to adjacent anatomical structures, problems may arise which, according to severity may force the patient to seek medical care. Aesthetics, hygiene, phonetics, mastication, swallowing and food flow pattern can be affected in a patient who has extensive or a large sized tori and/or exostoses. In this case since bony exostoses was small and had expanded uniformly the patient was not able to appreciate any disturbances. One of the reasons for such adaptation is the ability of the tongue to adapt immediately to unwanted stimulus within the oral cavity once the stimulus is persistent. Palatal exostoses in the location as described in this article has more significance in removable complete denture fabrication since the tissues over the tori tend to be thin and non resilient, which if not properly planned impairs denture stability. Whether they enhance or impair the oral stereognosis ability ${ }^{8}$ of a patient is a subject for research since no such research exists.

From a differential diagnostic point of view one should be able to differentiate the condition from conditions like osteoma (exosteal), subperiosteal hematoma, mature ossifying fibroma and early chondrosarcoma or osterosarcoma. ${ }^{9}$ The soft tissue overlying the bone may appear stretched and pale which can be mistaken to a white lesion in the area. When it interferes with normal functions, surgical removal should be considered. 
Bony Exostoses on Hard Palate - Prosthetic Inferences

\section{CONCLUSION}

Palatal exostoses are normal variations of bone and should be identified whenever a denture prosthesis is planned which covers the area. Under normal circumstances, the condition is inert and does not require any intervention since patients adapt well to such condition.

\section{ACKNOWLEDGEMENTS}

The authors would like to acknowledge the efforts of the staff or department of oral diagnosis for providing their valuable opinion about the case.

\section{REFERENCES}

[1] Fox J. The natural history and diseases of the teeth. London; 1814.

[2] Jainkittivong A, Langlais RP. Buccal and palatal Exostosis: prevalence and concurrence with tori. Oral Surg Oral Med Oral Pathol Oral Radiol Endod 2000; 90 (1): 48-53.

[3] Czuszak CA, Tolson GE, Kudryk VL, Hanson BS, Billman MA. Development of an exostosis following a free gingival graft: case report. J Periodontol 1996; 67 (3): 250-3.
[4] Mattoo KA, Kumar S. Mandibular tori-difficulties encountered during tooth preparation for a fixed partial denture. J Anat Sci 2009; 3: 26-28

[5] Kumar L, Mattoo KA, Singh K, Sahni S,Yadav A. Restoration of oronasal function in sub mucosal cleft by utilizing a palatal lift prosthesis. Clinical Dentistry 2012:26-30

[6] Seah YH. Torus palatinus and torus mandibularis: a review of the literature. Aust Dent J 1995; 40: 318-21

[7] Antoniades DZ, Belazi M, Papanayaiotou P. Concurrence of torus palatinus with palatal and buccal exostoses. Case report and review of the literature. Oral Surg Oral Med Oral Pathol Oral Radiol Endod 1998; 85 : 552-7

[8] Singh V, Mattoo KA. Oral Stereognosis pattern in patients with dental prosthesis in the elderly population. Int J Clin Exp Physiol 2014; 1(2): 211-5

[9] Chaudhry SI, Tappuni AR, Challacombe SJ. Multiple maxillary and mandibular exostoses associated with multiple dermatofibromas: a case report. Oral Surg Oral Med Oral Pathol Oral Radiol Endod 2000; 89(3): 319-22.

Citation: Shuja Rahman, Anchal Deep, Khurshid A Mattoo. Bony Exostoses on Hard Palate - Prosthetic Inferences. Archives of Dentistry and Oral Health. 2019; 2(2): 01-03.

Copyright: (c) 2019 Shuja Rahman, Anchal Deep, Khurshid A Mattoo. This is an open access article distributed under the Creative Commons Attribution License, which permits unrestricted use, distribution, and reproduction in any medium, provided the original work is properly cited. 